
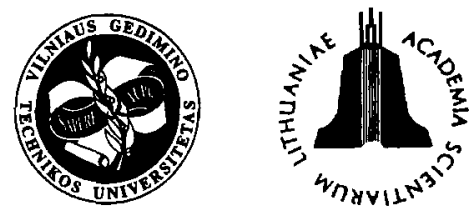

JOURNAL OF CIVIL ENGINEERING AND MANAGEMENT

http:/www.vtu.lt/english/edition

2002, Vol VIII, No 1, 49-53

\title{
FORMULATIONS OF THE DEFORMABLE FOUNDATION CONTACT PROBLEMS
}

\author{
Stanislovas Kalanta ${ }^{1}$, Andrius Grigusevičius ${ }^{2}$, Antanas Krutinis ${ }^{3}$ \\ Dept of Structural Mechanics, Vilnius Gediminas Technical University, \\ Saulètekio al. 11, LT-2040 Vilnius, Lithuania
}

Received 20 Feb 2001; accepted 15 Sept 2001

\begin{abstract}
Up to now in many works a construction on elastic foundation was modelled by the Winkler's hypothesis. In this paper the flexible plate-foundation system is investigated as an interaction of separate elastic bodies, which lie on the contact surface. Such a problem formulation allowed to make more exact the stress and strain distribution in the construction and the contact surface, to estimate the dead weight of the foundation. The presented numerical examples are realised using computer programme COSMOS/M. The obtained results show evidently the expediency of the new method application in practice.
\end{abstract}

Keywords: deformable foundation, variational formulations, finite element method.

\section{Introduction}

The solution of construction on elastic foundation can be formulated as a contact problem applying also the Winkler's hypothesis $[1,2,3]$. But solving the problem in this way will result in many shortages. It is necessary to point out some of them: it is imposible to estimate an influence of foundation settlements on the distribution of bending strains in case of a flexible plate (with evenly distributed loading) on deformable foundation analysis. Applying the Winkler's hypothesis we can get only a common settlement of flexible plate foundation without any bending strains. It is also impossible to estimate stress-strain distribution in the foundation depth, since in such problems the stress-strain state can be determined only in a contact surface between substracture and foundation. These contact problem solution shortages can be partialy eliminated using the elastic theory methods. However, only the elastic theory equations describing three-dimensional body stress-strain state do not fully reflect a real interaction between the construction and deformable foundation. Not only the construction but also the foundation are adopted as three-D bodies of finite dimensions. The settlements of the lower foundation surface are strictly limited, therefore the foundation does not correspond to the model of elastic halfspace.

The aim of this work is to define in a more exact way the stress-strain state by estimating not only ben-

\footnotetext{
E-mail: kal@st.vtu.lt

2 E-mail: a_grig@hotmail.com

3 E-mail: akr@st.vtu.lt
}

ding strains of a continuous construction, but also deformable foundation dead weight. The flexible platefoundation system is investigated in elastic state as an interaction of separate bodies. Such a formulation allows to go over from the contact problems solution to a synthesis of the elastic theory and these problems. General mathematical models are formulated here. They estimate the distribution of stresses and strains not only in the construction and contact surface, but also in the depth and width of the foundation. Mathematical models in this paper are realised using computer programme COSMOS/ M. Flexible plate stress-strain state is determined describing the flexible plate-foundation system by elements with different physical mechanical indices.

\section{Main equations}

Let's consider the stress-strain state of a system of two bodies (a plate and a deformable foundation). The system's construction volume is $V_{k}$ and the foundation volume is $V_{p}$ (Fig 1). The system surfaces are $S=S_{f} \cup S_{p} \cup S_{u}$, here $S_{u}$ is the contact surface between the construction and foundation, $S_{p}$ is border surfaces of the foundation. The system is affected by external loads described by vector function $\mathbf{q}(\mathbf{x}) \in S_{f}$, and vectors functions of the dead weight $\mathbf{g}_{k}(\mathbf{x}) \in V_{k}$, $\mathbf{g}_{p}(\mathbf{x}) \in V_{p}$.

Intensities and directions of the loading and dead weight are known. Stress-strain state of the construction is described by vectors functions $\sigma_{k}(\mathbf{x}) \in V_{k}, \mathbf{\varepsilon}_{k}(\mathbf{x}) \in V_{k}$, $\mathbf{u}_{k}(\mathbf{x}) \in V_{k}$. Stress-strain state of the deformable foundation is described by stress vector function $\sigma_{p}(\mathbf{x}) \in V_{p}$, 


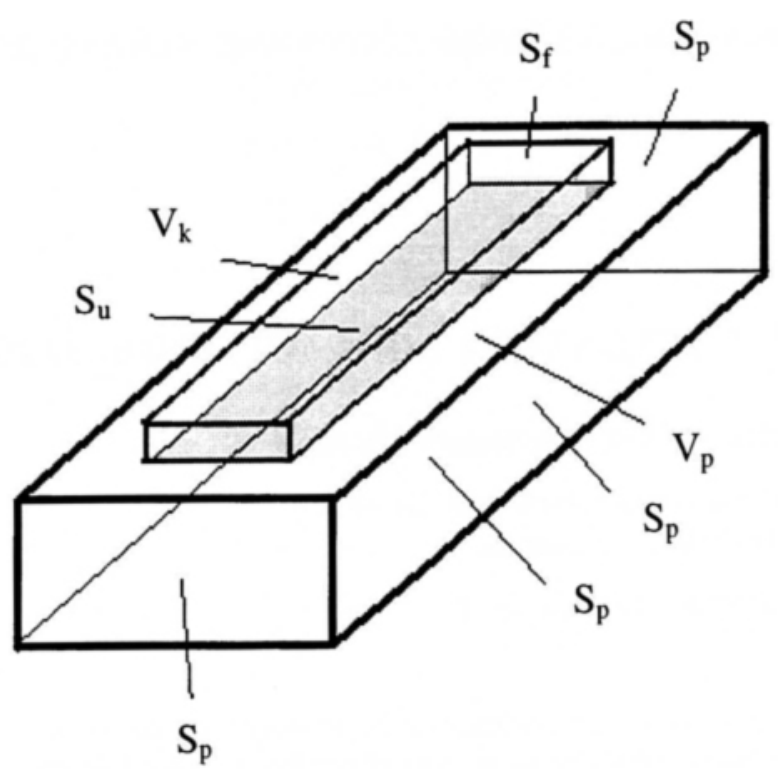

Fig 1. Flexible plate-foundation system

strain vector function $\varepsilon_{p}(\mathbf{x}) \in V_{p}$ and settlement vector function $\mathbf{s}(\mathbf{x}) \in V_{p}$.

Statically admissible stress vectors functions $\mathbf{\sigma}_{k}(\mathbf{x})$, $\boldsymbol{\sigma}_{p}(\mathbf{x})$ are described by static equations and static boundary conditions:

$$
\left.\begin{array}{cc}
-[A] \boldsymbol{\sigma}_{k}(\mathbf{x})=\mathbf{g}_{k}(\mathbf{x}) & \in V_{k}, \\
-[A] \boldsymbol{\sigma}_{p}(\mathbf{x})=\mathbf{g}_{p}(\mathbf{x}) & \in V_{p}, \\
{[N] \boldsymbol{\sigma}_{k}(\mathbf{x})=\mathbf{q}(\mathbf{x})} & \in S_{f}, \\
{[N] \boldsymbol{\sigma}_{k}(\mathbf{x})-\mathbf{p}(\mathbf{x})=\mathbf{0}} & \in S_{u}, \\
{[N] \boldsymbol{\sigma}_{p}(\mathbf{x})-\mathbf{p}(\mathbf{x})=\mathbf{0}} & \in S_{u}, \\
\mathbf{p}(\mathbf{x}) \leq \mathbf{0} & \in S_{u}, \\
{[N] \mathbf{\sigma}_{p}(\mathbf{x})-\mathbf{p}_{p}(\mathbf{x})=\mathbf{0}} & \in S_{p} .
\end{array}\right\}
$$

Here $\mathbf{p}(\mathbf{x})$ is the function of stresses in the contact surface $S_{u} ; \mathbf{p}_{p}(\mathbf{x})$ is the function of reaction pressures in the contact surface of the foundation $S_{p}$.

Kinematically admissible displacement and strain vectors functions $\mathbf{u}_{k}(\mathbf{x}), \mathbf{s}(\mathbf{x}), \varepsilon_{k}(\mathbf{x}), \boldsymbol{\varepsilon}_{p}(\mathbf{x})$ in the construction-foundation system are described by geometrical equations:

$$
\left.\begin{array}{ll}
{[A]^{T} \mathbf{u}_{k}(\mathbf{x})-\varepsilon_{k}(\mathbf{x})=\mathbf{0}} & \in V_{k}, \\
{[A]^{T} \mathbf{s}(\mathbf{x})-\varepsilon_{p}(\mathbf{x})=\mathbf{0}} & \in V_{p}, \\
\mathbf{u}_{k}(\mathbf{x})=\mathbf{s}(\mathbf{x}) & \in S_{u} .
\end{array}\right\}
$$

The connection between stress vectors functions $\mathbf{\sigma}_{k}(\mathbf{x}), \mathbf{\sigma}_{p}(\mathbf{x})$ and strain vectors functions $\varepsilon_{k}(\mathbf{x}), \varepsilon_{p}(\mathbf{x})$ of the construction-foundation system we describe by physical equations. These equations will be formulated for construction and elastic foundation:

$$
\left.\begin{array}{l}
{\left[D_{k}\right] \boldsymbol{\sigma}_{k}(\mathbf{x})=\varepsilon_{k}(\mathbf{x}) \in V_{k},} \\
{\left[D_{p}\right] \boldsymbol{\sigma}_{p}(\mathbf{x})=\varepsilon_{p}(\mathbf{x}) \in V_{p} .}
\end{array}\right\}
$$

Here $\left[D_{k}\right],\left[D_{p}\right]$ are flexibility matrices of physical equations for the construction and foundation respectively, which depend on modulus of elasticity $E, E_{0}$ and Poisson's ratios $v, v_{0}$ :

Boundary conditions (3) must be satisfied at the foundation border surface $S_{p}$ :

$$
[D] \mathbf{p}_{p}(\mathbf{x})+\mathbf{s}(\mathbf{x})=\mathbf{0} \in S_{p},
$$

Here $[D]$ is the foundation flexibility matrix, the diagonal elements of which are inversely proportional to stiffness ratio $C$.

\section{Full equation system to define the actual stress- strain state}

Static equilibrium, geometrical and physical equations and boundary conditions (1)-(4) form a full system of equations for flexible plate and foundation. This system describes elastic stress-strain state of the construction and foundation. We can eliminate strains $\varepsilon_{k}(\mathbf{x})$, $\varepsilon_{p}(\mathbf{x})$ and stresses $\mathbf{p}(\mathbf{x})$ from (1)-(4) equations:

$$
\begin{array}{ll}
-[A] \boldsymbol{\sigma}_{k}(\mathbf{x})=\mathbf{g}_{k}(\mathbf{x}) & \in V_{k}, \\
-[A] \boldsymbol{\sigma}_{p}(\mathbf{x})=\mathbf{g}_{p}(\mathbf{x}) & \in V_{p}, \\
{\left[D_{k}\right] \boldsymbol{\sigma}_{k}(\mathbf{x})-[A]^{T} \mathbf{u}_{k}(\mathbf{x})=\mathbf{0}} & \in V_{\mathbf{k}}, \\
{\left[D_{p}\right] \boldsymbol{\sigma}_{p}(\mathbf{x})-[A]^{T} \mathbf{s}(\mathbf{x})=\mathbf{0}} & \in V_{p}, \\
{[D] \mathbf{p}_{p}(\mathbf{x})+\mathbf{s}(\mathbf{x})=\mathbf{0}} & \in S_{p}, \\
{[N] \boldsymbol{\sigma}_{k}(\mathbf{x})=\mathbf{q}(\mathbf{x})} & \in S_{f}, \\
{[N] \boldsymbol{\sigma}_{k}(\mathbf{x})-[N] \boldsymbol{\sigma}_{p}(\mathbf{x})=\mathbf{0}} & \in S_{u}, \\
\mathbf{u}_{k}(\mathbf{x})-\mathbf{s}(\mathbf{x})=\mathbf{0} & \in S_{u}, \\
\mathbf{p}(\mathbf{x}) \leq \mathbf{0} & \in S_{u} .
\end{array}
$$

The stress-strain state of flexible plate and elastic foundation in this mathematical model is described by:

- static equilibrium equations for the construction (5) and foundation (6);

- geometrical equations for the construction (7) and foundation (8);

- boundary conditions (9) of the foundation surface $S_{p}$

- static boundary conditions (10) of the construction surface $S_{f}$;

- static and kinematic compatibility conditions (11), (12) of the construction and foundation contact surface $S_{u}$

- condition (13) limiting the sign of the reaction pressures of the foundation.

\section{Variational formulations of the problem}

Usually one or another kind of variational formulations is used to solve the elastic theory problems by finite element method. Direct solution of the system of equations (5)-(13) is too complicated. Some variational formulations of such a problem can be produced using 
total complementary energy (Castigliano's) principle [4, 5]:

Of all statically possible stresses fields, the one that total complementary energy of system is minimal is the true one.

Total complementary energy of the system is:

$$
\begin{aligned}
& U=\frac{1}{2} \int_{V_{k}} \boldsymbol{\sigma}_{k}^{T}(\mathbf{x})\left[D_{k}\right] \boldsymbol{\sigma}_{k}(\mathbf{x}) d V_{k}+ \\
& +\frac{1}{2} \int_{V_{p}} \sigma_{p}^{T}(\mathbf{x})\left[D_{p}\right] \boldsymbol{\sigma}_{p}(\mathbf{x}) d V_{p}+\frac{1}{2} \int_{S_{p}}^{T} \mathbf{p}_{p}^{T}(\mathbf{x})[D] \mathbf{p}_{p}(\mathbf{x}) d S_{p},
\end{aligned}
$$

and statically possible fields of stresses are described by the system of equations (1). Therefore, by Castigliano's principle we can get such a static variational formulation of the problem:

$$
\begin{aligned}
& \frac{1}{2} \int_{V_{k}} \boldsymbol{\sigma}_{k}^{T}(\mathbf{x})\left[D_{k}\right] \boldsymbol{\sigma}_{k}(\mathbf{x}) d V_{k}+\frac{1}{2} \int_{V_{p}} \boldsymbol{\sigma}_{p}^{T}(\mathbf{x})\left[D_{p}\right] \boldsymbol{\sigma}_{p}(\mathbf{x}) d V_{p}+ \\
& +\frac{1}{2} \int_{S_{p}} \mathbf{p}_{p}^{T}(\mathbf{x})[D] \mathbf{p}_{p}(\mathbf{x}) d S_{p} \Rightarrow \min
\end{aligned}
$$

when

$$
\left.\begin{array}{cc}
-[\mathrm{A}] \boldsymbol{\sigma}_{k}(\mathbf{x})=\mathbf{g}_{k}(\mathbf{x}) & \in V_{k}, \\
-[\mathrm{A}] \boldsymbol{\sigma}_{p}(\mathbf{x})=\mathbf{g}_{p}(\mathbf{x}) & \in V_{p}, \\
{[\mathrm{~N}] \boldsymbol{\sigma}_{k}(\mathbf{x})=\mathbf{q}(\mathbf{x})} & \in S_{f}, \\
{[\mathrm{~N}] \boldsymbol{\sigma}_{k}(\mathbf{x})-\mathbf{p}(\mathbf{x})=\mathbf{0}} & \in S_{u}, \\
{[\mathrm{~N}] \boldsymbol{\sigma}_{p}(\mathbf{x})-\mathbf{p}(\mathbf{x})=\mathbf{0}} & \in S_{u}, \\
\mathbf{p}(\mathbf{x}) \leq \mathbf{0} & \in S_{u}, \\
{[\mathrm{~N}] \boldsymbol{\sigma}_{p}(\mathbf{x})-\mathbf{p}_{p}(\mathbf{x})=\mathbf{0}} & \in S_{p}
\end{array}\right\}
$$

This formulation allows to determine the distribution of stresses in the volume of the construction, foundation and reaction pressures $\mathbf{p}_{p}(\mathbf{x})$ in the foundation surface $S_{p}$.

Using Lagrangian multiplier method we can make a dual formulation of the problem:

$$
\begin{aligned}
& -\frac{1}{2} \int_{V_{k}} \boldsymbol{\sigma}_{k}^{T}(\mathbf{x})\left[D_{k}\right] \boldsymbol{\sigma}_{k}(\mathbf{x}) d V_{k}-\frac{1}{2} \int_{V_{p}} \boldsymbol{\sigma}_{p}^{T}(\mathbf{x})\left[D_{p}\right] \boldsymbol{\sigma}_{p}(\mathbf{x}) d V_{p}- \\
& -\frac{1}{2} \int_{S_{p}} \mathbf{p}_{p}^{T}(\mathbf{x})[D] \mathbf{p}_{p}(\mathbf{x}) d S_{p}+\int \mathbf{u}_{V_{k}}^{T}(\mathbf{x}) \mathbf{g}_{k}(\mathbf{x}) d V_{k}+ \\
& +\int_{V_{p}} \mathbf{s}^{T}(\mathbf{x}) \mathbf{g}_{p}(\mathbf{x}) d V_{p}+\int_{S_{f}} \mathbf{u}_{k}^{T}(\mathbf{x}) \mathbf{q}_{k}(\mathbf{x}) d S_{f} \Rightarrow \max (16) \\
& \text { when }
\end{aligned}
$$

$$
\left.\begin{array}{ll}
{\left[\mathrm{D}_{\mathrm{k}}\right] \boldsymbol{\sigma}_{k}(\mathbf{x})-[\mathrm{A}]^{\mathrm{T}} \mathbf{u}_{k}(\mathbf{x})=\mathbf{0}} & \in V_{k}, \\
{\left[\mathrm{D}_{\mathrm{p}}\right] \boldsymbol{\sigma}_{p}(\mathbf{x})-[\mathrm{A}]^{\mathrm{T}} \mathbf{s}(\mathbf{x})=\mathbf{0}} & \in V_{p}, \\
{[\mathrm{D}] \mathbf{p}_{p}(\mathbf{x})+\mathbf{s}(\mathbf{x})=\mathbf{0}} & \in S_{p}, \\
\mathbf{u}_{k}(\mathbf{x})-\mathbf{s}(\mathbf{x}) \leq \mathbf{0} & \in S_{\mathbf{u}}
\end{array}\right\}
$$

The conditions of this problem describe a lot of $\mathrm{ki}$ nematically admissible displacement fields of the construction and settlement fields of the foundation. The objective function multiplied by -1 expresses the total potential energy of the deformable system. Therefore the extreme problem (16)-(17) expresses Langrangian principle:

Of all kinematically possible displacement fields, the one that total potential energy of deformable system is minimal is the true one.

This mathematical model allows to determine stresses and displacements of the system directly, but it is not convenient, because it includes many unknowns. We can simplify it by eliminating stress functions. From the equation system (17) we derive:

$$
\left.\begin{array}{ll}
\boldsymbol{\sigma}_{k}(\mathbf{x})=\left[D_{k}\right]^{-1}[A]^{T} \mathbf{u}_{k}(\mathbf{x}) & \in V_{k}, \\
\boldsymbol{\sigma}_{p}(\mathbf{x})=\left[D_{p}\right]^{-1}[A]^{T} \mathbf{s}(\mathbf{x}) & \in V_{p}, \\
\mathbf{p}_{p}(\mathbf{x})=-[D]^{-1} \mathbf{s}(\mathbf{x}) & \in S_{p} .
\end{array}\right\}
$$

Putting these expressions of stress functions into objective function (16) such a kinematic formulation of the problem is derived:

$$
\begin{aligned}
& -\frac{1}{2} \int_{V_{k}}\left\{[A]^{T} \mathbf{u}_{k}(\mathbf{x})\right\}^{T}\left[D_{k}\right]^{-1}[A]^{T} \mathbf{u}_{k}(\mathbf{x}) d V_{k}- \\
& -\frac{1}{2} \int_{V_{p}}\left\{[A]^{T} \mathbf{s}(\mathbf{x})\right\}^{T}\left[D_{p}\right]^{-1}[A]^{T} \mathbf{s}(\mathbf{x}) d V_{p}- \\
& -\frac{1}{2} \int_{S_{p}} \mathbf{s}^{T}(\mathbf{x})[D]^{-1} \mathbf{s}(\mathbf{x}) d S_{p}+\int_{V_{k}} \mathbf{u}_{k}^{T}(\mathbf{x}) \mathbf{g}_{k}(\mathbf{x}) d V_{k}+ \\
& +\int_{V_{p}} \mathbf{s}^{T}(\mathbf{x}) \mathbf{g}_{p}(\mathbf{x}) d V_{p}+\int_{S_{f}} \mathbf{u}_{k}^{T}(\mathbf{x}) \mathbf{q}_{k}(\mathbf{x}) d S_{f} \Rightarrow \max
\end{aligned}
$$

when

$$
\mathbf{u}_{k}(\mathbf{x})-\mathbf{s}(\mathbf{x}) \leq \mathbf{0} \quad \in S_{u} .
$$

The unknowns of this problem are only kinematic values: displacements of the construction $u_{k}(x)$ and settlements of the foundation $\mathbf{s}(\mathbf{x})$.

The second mathematical model (19)-(20) is easier than the first one to realise by the finite element method. Moreover, we can use many widespread computer programs for such problems. Using them, the problem (19)-(20) can be solved by iterative method, ie by changing the equation (20) into the equation

$$
\mathbf{u}_{k}(\mathbf{x})-\mathbf{s}(\mathbf{x})=\mathbf{0} \quad \in S_{u} .
$$

If after one iteration in some construction and foundation contact zones tension stresses are derived, then in the next iteration the problem is formulated without contact conditions (21) in these zones.

We solve the flexible plate-foundation system by finite element method using geometrically compatible finite elements and taking principal unknowns in the finite elements nodes. 
Displacement approximative functions are set for $j$-th finite element of plate and foundation:

$$
\mathbf{u}_{k j}(\mathbf{x})=\left\lfloor H_{k j}(\mathbf{x})\right\rfloor \mathbf{u}_{k j} ; \quad \mathbf{s}_{j}(\mathbf{x})=\left\lfloor H_{p j}(\mathbf{x})\right\rfloor \mathbf{s}_{j},
$$

here $\mathbf{u}_{k j}, \mathbf{s}_{j}$ are displacements vectors of $j$-th finite element nodes of plate and foundation.

Estimating these functions for finite element model, (19) a functional can be expressed:

$$
\begin{aligned}
& -\sum_{j=1}^{n_{k}} \frac{1}{2} \int_{V_{k j}}\left\{|A|^{T}\left[H_{k j}(\mathbf{x})\right] \mathbf{u}_{k j}\right\}^{T}\left[D _ { k } | ^ { - 1 } \left[\left.A\right|^{T}\left[H_{k j}(\mathbf{x})\right] \mathbf{u}_{k j} d V_{k j}-\right.\right. \\
& -\sum_{j=1}^{n_{p}} \frac{1}{2} \int_{V_{p j}}\left\{|A|^{r}\left[H_{p j}(\mathbf{x})\right] \mathbf{s},\right\}\left[D_{j}\right\}^{-1}|A|^{T}\left[H_{p j}(\mathbf{x})\right] \mathbf{s}, d V_{p j}- \\
& -\sum_{j=1}^{n_{j}} \frac{1}{2} \int_{S_{p j}} \mathbf{s}_{j}^{T}\left[H_{p j}(\mathbf{x})\right]^{T}\left[\left.D\right|^{-1}\left[H_{p j}(\mathbf{x})\right] \mathbf{s}_{j} d S_{p j}+\right. \\
& +\sum_{j=1 V_{k j}}^{n_{k}} \int_{V_{k j}} \mathbf{u}_{k j}^{T}\left[H_{k j}(\mathbf{x})\right]^{T} \mathbf{g}_{k j}(\mathbf{x}) d V_{k j}+\sum_{j=1 V_{p j}}^{n_{p}} \mathbf{s}_{j}^{T}\left[H_{p j}(\mathbf{x})\right]^{T} \mathbf{g}_{p j}(\mathbf{x}) d V_{p j}+ \\
& +\sum_{j=1}^{n_{k}} \int_{S_{j}} \mathbf{u}_{k j}^{T}\left[H_{k j}(\mathbf{x})\right]^{T} \mathbf{q}_{k j}(\mathbf{x}) d S_{j j}=F
\end{aligned}
$$

here $n_{k}, n_{p}$ are the number of finite elements of the plate and the foundation respectively; $n_{s}$ is boundary elements number of the foundation in the surface $S_{p}$. Expressing this functional by global discrete model displacements $\mathbf{u}_{k}, \mathbf{s}$, estimating the connections of them with finite element displacements, expressing stationary conditions of the functional and displacements compatibility equations (21) in the plate-foundation contact surface we derive such a system of equations:

$$
\begin{aligned}
& {\left[K_{k}\right] \mathbf{u}_{k}=\mathbf{F}_{k},} \\
& {\left[K_{p}\right] \mathbf{s}=\mathbf{F}_{p},} \\
& {\left[A_{k}\right]\left(\mathbf{u}_{k}-\mathbf{s}\right)=\mathbf{0} .}
\end{aligned}
$$

The vector $\mathbf{s}$ of foundation settlements can be divided into two parts $\mathbf{s}=\left\{\mathbf{s}_{k}, \mathbf{s}_{p}\right\}^{T}$, here $\mathbf{s}_{k}$ is the displacements of finite elements nodes in the surface $S_{u} ; \mathbf{s}_{p}$ is the displacements of foundation elements nodes that do not belong to the contact surface. Then, using contact conditions, we can eliminate displacements $\boldsymbol{s}_{k}$ from (23) equation system and get traditional finite elements equation:

$$
[K] \mathbf{u}=\mathbf{F},
$$

here $\mathbf{u}=\left\{\mathbf{u}_{k}, \mathbf{s}_{p}\right\}^{T}$ is the vector of plate foundation finite elements nodes displacements; $\mathbf{F}$ is the vector of discrete model nodal forces, derived combining the vectors of nodal forces vectors $\mathbf{F}_{k}$ and $\mathbf{F}_{p}$. Then nodal displacements

$$
\mathbf{u}=[K]^{-1} \mathbf{F},
$$

and finite elements stresses of the system

$$
\begin{aligned}
& \boldsymbol{\sigma}_{k j}(\mathbf{x})=\left[D_{k}\right]^{-1}[A]^{T}\left[H_{k j}(\mathbf{x})\right] \mathbf{u}_{k j}, \\
& \boldsymbol{\sigma}_{p j}(\mathbf{x})=\left[D_{p}\right\}^{-1}[A]^{T}\left[H_{p j}(\mathbf{x})\right] \mathbf{s}_{j} .
\end{aligned}
$$

\section{Numerical example}

The efficiency of the presented method will be illustrated by two solutions of analysis problem of deformable foundation. The foundation of discrete model in the first numerical case (Fig 2) is estimated as a volu-

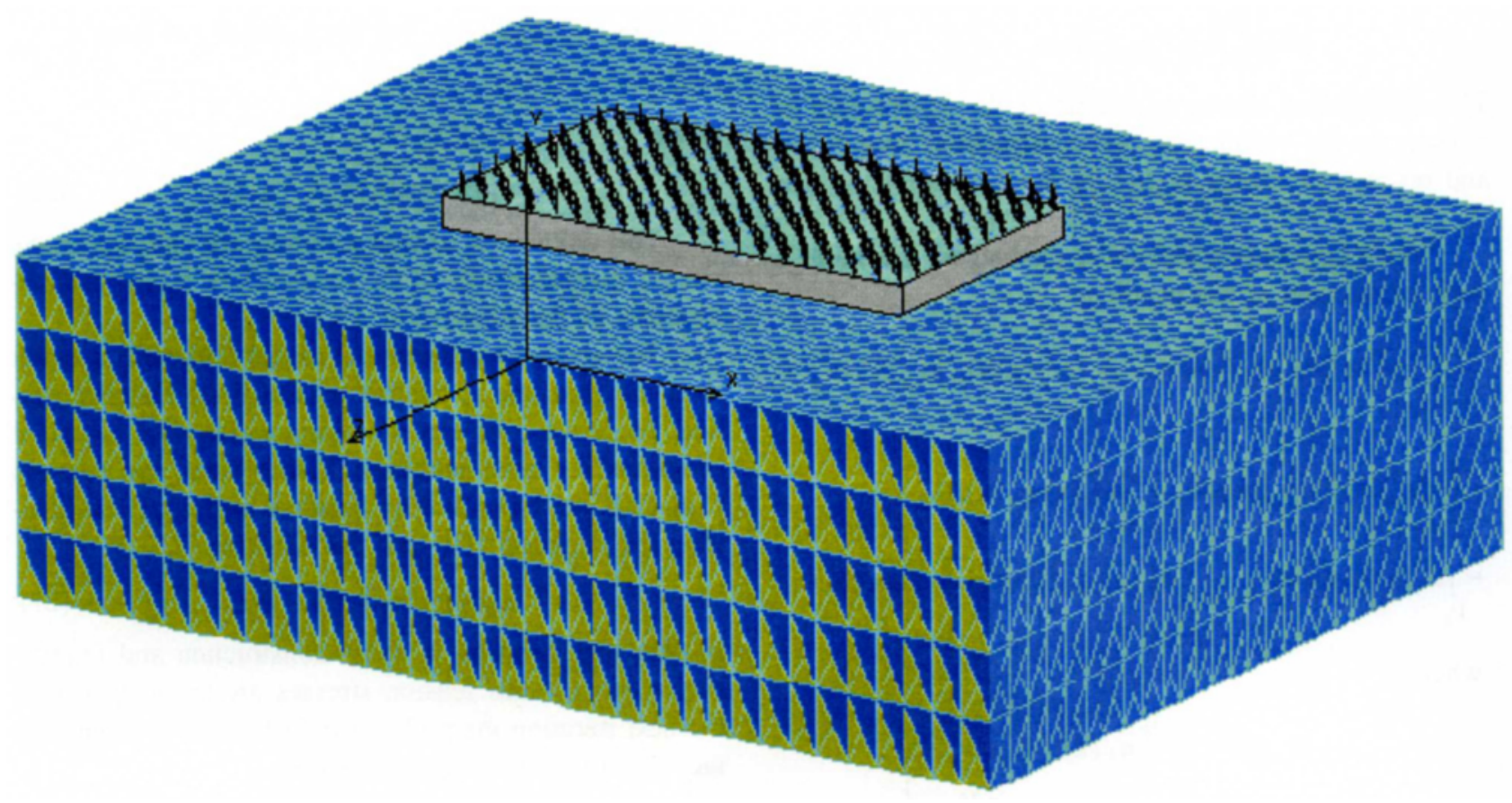

Fig 2. Finite elements and loading of plate foundation system 
metric body of parallelepiped rectangle shape that is bounded from below and sides by finite thickness layers modelled by the Winkler's hypothesis with stiffness ratio $C_{1}$. The foundation is continuous, ie has the same physical mechanical indices. In the discrete model of the second problem the entire foundation is estimated as a finite thickness layer deformed according to the Winkler's hypothesis with stiffness ratio $C_{2}$. The rest data are the same for both cases.

Flexible plate: length $8 \mathrm{~m}$, width $4.5 \mathrm{~m}$, thickness $0.4 \mathrm{~m}$, density $2100 \mathrm{~kg} / \mathrm{m}^{3}$, modulus of elasticity $7000000 \mathrm{kPa}$, Poisson's ratio 0.2 , discretization 4-node tetrahedron solid finite element type, loading constant uniformly distributed load $90 \mathrm{kN} / \mathrm{m}^{2}$ acts on the top surface of the plate.

Foundation: length $17 \mathrm{~m}$, width $13.5 \mathrm{~m}$, height $4.5 \mathrm{~m}$, density of the soil $1600 \mathrm{~kg} / \mathrm{m}^{3}$, modulus of elasticity $40000 \mathrm{kPa}$, Poisson's ratio 0.3 , discretization 4-node tetrahedron solid finite element type, foundation stiffness ratio:

for the first discrete model

$$
C_{1}=\frac{E_{0}}{\left(1-v_{0}\right)^{2} H_{l a 1}}=\frac{40000}{(1-0.3)^{2} \cdot 6.75}=12094 \mathrm{kPa} / \mathrm{m}
$$

for the second discrete model

$$
C_{2}=\frac{E_{0}}{\left(1-v_{0}\right)^{2} H_{l a 2}}=\frac{40000}{(1-0.3)^{2} \cdot(4.5+6.75)}=7256 \mathrm{kPa} / \mathrm{m} \text {; }
$$

here $E_{0}$ is the modulus of elasticity of the soil; $v_{0}$ is Poisson's ratio of the soil; $H_{l a l}, H_{l a 2}$ are deformable layer thickness of the foundation for the first and second discrete model respectively.

Deformable layer thickness of the foundation for the first problem is equal to the half width of the foundation as a volumetric body $H_{l a 1}=13.5 / 2=6.75 \mathrm{~m}$. In the second problem, the lower bound of deformable layer is at the same level as in the first problem, therefore

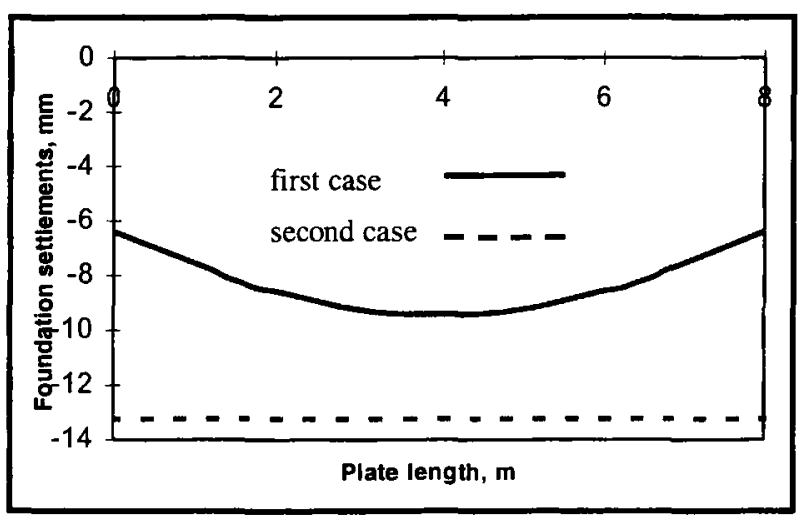

Fig 3. Distribution of foundation settlements
$H_{l a 2}=4.5+6.75=11.25 \mathrm{~m} . \mathrm{COSMOS} / \mathrm{M}$ computer programme [6] is used to solve these problems.

The obtained results (Fig 3) show that the foundation settlement in the first case corresponds to the real surface of the construction deflection. Meanwhile, in the second case all the settlements of the foundation are the same, ie a plane corresponds to the deformable surface of the flexible plate. The maximum settlement of the foundation in the first case is by $29 \%$ less than in the second discrete model.

\section{Conclusions}

1. The presented problem of construction on elastic foundation, or the problem of an interaction between flexible plate and foundation evidently shows that it is reasonable to go over from a contact solving such a type problem to estimating the stress-strain state by the elastic theory method.

2. Formulated mathematical models estimate not only a common stress-strain state of the constructionfoundation system, but also reflect the actual distribution of bending strains in the flexible plate.

3. The obtained results (the first case) reveal possibilities to estimate not only the distribution of stresses and displacements in the construction, but also the distribution of settlements and reaction pressures in the width, length and depth of the foundation.

4. The presented method allows to estimate more exactly the influence of dead weight on stress and strain distribution not only in the construction but also in the soil foundation.

\section{References}

1. Lancellotta $R$. Geotechnical engineering. Technical University of Turin, Italy, 1995. $448 \mathrm{p}$.

2. Aleynikov S. M. \& Ikonin S. V. Numerical modeling of contact interaction of bases with dredging wells of bridge piers. Proc. of the 12th European conf. „, Geotechnical engineering for transportation infrastructure". A. A. Balkema, Amsterdam, 1999, Vol 3, p. 1719-1724.

3. Ortigao J. A. R. Soil mechanics in the light of critical state theories: An introduction. Federal University Rio de Janeiro, Brazil, 1995. 160 p.

4. Krutinis A. Mathematical models for design of elastic structures on deformable ground. Lithuanian Journal of Computational Mechanics, Vol 33, 1993, p. 108-116.

5. Kalanta S. Relations and transformations of extremum energy principles for deformable body. Statyba (Civil Engineering). Nr. 1(9). Vilnius: Technika, 1997, p. 49-64.

6. COSMOS/M. Comand reference. Structural Research and Analysis Corporation. Los Angeles, California. Copyright 2000. $883 \mathrm{p}$ 